Revistade
Economid
Contemporâned

\title{
DETERMINANTES MACROECONÔMICOS DAS VENDAS NO VAREJO NO PERÍODO 2004-2011
}

\author{
Gustavo Inácio de Moraes ${ }^{a}$ \\ Thiago Leal Flores da Silva ${ }^{b}$

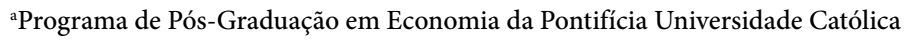 \\ do Rio Grande do Sul (PUC-RS). \\ ${ }^{\mathrm{b}}$ Banco do Brasil.
}

Artigo recebido em 19/06/2013 e aceito em 26/01/2015.

RESUMO: O presente artigo utiliza-se de uma estimação em painel, considerando-se seis estados brasileiros, para verificar a influência sobre as vendas no varejo de três componentes macroeconômicos, tais como a renda real média, a taxa de desemprego e a taxa de juros, entre os anos de 2004 e 2011, período no qual a economia brasileira observou expressivo crescimento econômico. Optou-se por uma estimação em SUR (regressões aparentemente não correlacionadas) para ampliar o ajuste ao longo dos espaços geográficos estudados. Como resultado geral, nota-se que as vendas ao varejo são elásticas, contemporaneamente, à renda real média. As demais variáveis, taxa de desemprego e taxa de juros demonstraram-se inelásticas e em alguns estados sequer significativas, além de se manifestarem com uma defasagem de três meses.

PALAVRAS-CHAVE: Vendas no varejo; atividade econômica; economias estaduais.

CLASSIFICAÇÃO JEL: C23; E21; R11. 


\title{
MACROECONOMIC DETERMINANTS OF RETAIL SALES IN BRAZIL, 2004-2011
}

\begin{abstract}
This paper performs a panel exercise, including six Brazilian states, to determine the influence on retail sales of three main macro-indicators, such as personal income, unemployment rate and interest rates, in the 2004-2011period, when Brazilian economy showed an expressive economic growth rate. A SUR (seemingly uncorrelated residuals) methodology estimation across Brazilian states was conducted. All results point out that retail sales and personal income are linked contemporarily and, at the same time, elastic. As for unemployment rate and interest rates, despite their significance in most states, both are inelastic to explain retail sales and lagging three months in the macroeconomic context.
\end{abstract}

KEYWORDS: Retail sales; economic activity; regional economies. 


\section{INTRODUÇÃO}

$\mathrm{Na}$ evolução das economias modernas, entende-se que a predominância do setor de serviços no produto poderia representar um sinal de avanço econômico. Em grande parte, significaria que a economia atingiu um estágio no qual o consumo da sociedade seria responsável pela dinâmica da economia. Rostow (1970) expressava, por exemplo, que o estágio final do desenvolvimento econômico envolveria a "era do consumo em massa" como resultado da evolução econômica de quatro estágios anteriores.

No setor de serviços, pela classificação do IBGE (2011b), podemos encontrar diversos subsetores componentes do produto interno bruto (PIB), dentre os quais se destacam transporte, serviços de informação, setor financeiro, serviços imobiliários, educação e saúde pública, administração pública, outros serviços e comércio. Nesse particular, o setor de serviços responderia por $64 \%$ do PIB e o comércio, por $10,7 \%$ do total no ano de 2005. O subsetor de comércio, porém, é dividido em vendas no varejo, vendas no atacado e comércio de peças e automóveis, segundo a classificação apresentada por IBGE (2010b).

A importância do setor de comércio também pode ser notada pela quantidade de empregados no setor. Segundo IBGE (2010b), um total de 8,8 milhões de pessoas tem o setor como seu foco de atividade principal. Desses, 858 mil atuavam em comércio de veículos e peças; 1,5 milhão, em comércio por atacado; e 6,5 milhões, no comércio varejista. Ademais, enquanto o setor comércio faturou R\$ 59 bilhões em 2009, o comércio varejista obteve R $\$ 18$ bilhões, ou $30 \%$ do total.

O presente artigo tem como objeto de estudo especificamente o setor varejista, cuja empregabilidade é expressiva, bem como seu faturamento, indicando a extensão deste. O período de análise estende-se de janeiro de 2004 a junho de 2011, momento no qual a economia brasileira cresceu expressivamente, exceção feita ao ano de 2009. Nesse período, por consequência da política econômica, a renda pessoal real elevou-se, a taxa de juros na maior parte do período reduziu-se e a política fiscal foi ativa e, em paralelo, não houve pressão expressiva sobre o balanço de pagamentos. Portanto, essa conjunção de condições favoráveis tornou possível a expansão do crédito e, por extensão, do consumo pessoal.

Nosso objetivo é responder de que maneira as vendas no varejo são explicadas, no período, por variáveis macroeconômicas como renda, emprego e juros. Como objetivo específico, pretendemos determinar se há diferenças significativas entre esses padrões nos principiais estados brasileiros, especificamente Bahia, Minas Gerais, Pernambuco, Rio de Janeiro, Rio Grande do Sul e São Paulo. A escolha destes é justificada em função do dinamismo econômico destes estados, representado neste trabalho pela expressiva parcela de participação na economia. Conforme Vasconcelos et al. (2004), em agosto 
de 1994 São Paulo possuía 53,68\% do total de crédito contratado no Brasil, frente a 10,45\% do Rio de Janeiro, e 6,60\%, 5,59\%, 6,33\% e 3,65\% de Minas Gerais, Rio Grande do Sul, Paraná e Bahia, respectivamente.

Para o alcance dos objetivos, o artigo possui, além desta introdução, uma segunda seção, na qual há uma revisão sobre as estimações anteriores relacionadas às vendas no varejo; uma terceira seção, na qual a metodologia e os resultados são demonstrados; e, também, uma quarta seção onde comentários conclusivos são elaborados.

\section{VENDAS NO VAREJO E RESULTADOS ANTERIORES}

A importância do setor do varejo justificou a elaboração de diversos estudos relativos à previsibilidade das atividades do setor e seu comportamento frente às variações nos agregados macroeconômicos. Várias metodologias para esta finalidade foram aplicadas, de modo que se faz necessário situar como a metodologia que será utilizada nesse artigo se situa neste debate.

Alguns trabalhos exploram a previsão microeconômica das vendas no varejo e a influência de aspectos relativos aos consumidores. Outros trabalhos, no entanto, dedicam-se ao estudo do setor varejo em nível macroeconômico, sobretudo quando considerado o setor varejo um importante indicador antecedente.

No caso da decisão do consumidor dentro da loja ou confrontado a uma série de produtos, Almeida e Passari (2006) almejam antecipar os movimentos das vendas no varejo através da técnica de redes neurais. Os autores, principalmente, decompõem as vendas em suas diversas categorias e concluem que a técnica de redes neurais é adequada para quando se tem a possibilidade de desagregação das séries. Os autores destacam, sobretudo, que a técnica de redes neurais enquadra-se em um conjunto denominado data mining, onde os padrões e as correlações das séries são pesquisados e incorporados como informações à construção das tendências futuras. Assim, comparando-se a técnicas de regressão linear, por exemplo, as redes neurais mostram-se mais adequadas, mas, principalmente, sua eficiência como previsor de movimentos ocorre em curto prazo.

Em outra vertente, Nogueira, Deos e Brito (2011), ao analisarem os mecanismos de formação de preços do varejo, entendem que ideal seria uma combinação dos fatores microeconômicos e macroeconômicos, em especial destacando os riscos que a adoção de um sistema de metas para inflação produz em perspectiva da formação de preços no setor varejo.

Por outro lado, no nível macroeconômico, Araujo (2010), em especial, destaca que o amplo do crescimento do crédito no Brasil a partir de 2003 é um fenômeno notável. Em particular, destaca os fatores fundamentais para esta retomada do crédito que 
estariam fundamentados em políticas de crédito consignado, reduções constantes na taxa de juros e um ambiente macroeconômico favorável. Nesse sentido, pode-se sugerir que seu impacto sobre as vendas no varejo seja relevante.

Bronzi e Scarpel (2010) sugerem como método preditivo da atividade econômica em geral, especificamente o PIB, a composição de um grupo de atores representativos nos setores de atividade econômica. Assim, o modelo sugerido parte de uma diferenciação entre produção industrial e volume de venda no varejo. Finalmente, decompõe-se nos setores mais representativos de cada um desses grandes blocos. Então, os clusters de cada atividade são avaliados e através deles são obtidas as trajetórias possíveis para cada série. Segundo os autores, a técnica mostrou-se promissora, contudo, alertam que, quando da ocorrência da crise de 2008/2009, os dados subitamente perderam ajuste, o que nos dá margem a interpretar que o modelo não é capaz de identificar mudanças bruscas na mesma intensidade dos índices oficiais. Capitani et al. (2010) acrescentam que, a partir de organização de dados hierárquicos, quatro séries essenciais podem mostrar grande parte da situação econômica: produção intensiva em capital, produção para consumo, insumos para produção e consumo, sendo este último especialmente ligado às vendas no varejo.

Aproximando-se do objetivo deste artigo, Campos (2009) analisa os impactos da conjuntura macroeconômica sobre as vendas no varejo. Seu estudo, contudo, se concentra nos resultados econômico-financeiros de grandes corporações do setor varejista. É importante, porém, notar que são realizados exercícios econométricos nos quais as variáveis dependentes são índices de desempenho das firmas, tais como o faturamento bruto e variáveis independentes dos principais indicadores macroeconômicos, em frequência trimestral. Em geral, notou-se uma relação importante entre o faturamento das grandes redes varejistas com variáveis como renda e taxa de juros. Curiosamente, contudo, o crédito não se mostrou uma variável significativa para quaisquer exercícios.

Para Angelo et al. (2011), o varejo consiste precisamente no vínculo que se estabelece entre a indústria e o consumidor final. Prever as vendas é essencial para que se possa gerenciar de modo adequado os processos produtivos e de comercialização. No varejo, esse aspecto reveste-se de importância ainda maior. Vender significa harmonizar os interesses dos que produzem com os desejos daqueles que compram. Assim, Angelo et al. (2011) desenvolvem um modelo que consiste em examinar comparativamente a aplicação de dois métodos de previsão de vendas de varejo no mercado brasileiro: as séries temporais e as redes neurais. Embora a utilização de redes neurais tenha proporcionado bons resultados preditivos, os resultados empregando modelos do tipo Auto Regressive Integrated Moving Average (ARIMA) mostraram-se praticamente equivalentes. O período de comparação estende-se de julho de 2007 a junho de 2008, 
a partir de um modelo gerado entre junho de 2000 e junho de 2007. Ademais, outra técnica temporal, o alisamento exponencial, tanto na sua vertente multiplicativa quanto na vertente aditiva, demonstrou resultados inferiores às demais.

O sentido dos resultados obtidos também é confirmado por Pasquotto (2010), que se utiliza das Redes de Elman, ou redes neurais recorrentemente simples, para três séries de previsão de segmentos das vendas no varejo: o setor farmacêutico, adubos e tráfego aéreo. Os resultados, quando comparados à metodologia Box-Jenkins, demonstraram-se inferiores, mas, observada a correção para sazonalidade - movimento oscilatório recorrente, de periodicidade homogênea - e estacionariedade da série, a previsão pode ser aperfeiçoada. Nos dados com pré-processamento aconteceu a eliminação da tendência e da sazonalidade por meio das diferenças, surtindo um efeito benéfico na qualidade das previsões com o uso das redes de Elman. Este benefício não se mostrou unânime e encontrou exceção no caso da previsão dinâmica da série Adubos. De uma maneira geral, as previsões das três séries da pesquisa de Pasquotto (2010) com as séries de Elman, não superaram as previsões obtidas por meio de modelo lineares via metodologia de Box-Jenkins. Segundo o autor, a previsão com a rede de Elman foi apenas ligeiramente superior em um único caso entre as três séries testadas - Aéreo -, e com pouca vantagem comparativa.

Por outro lado, Faleiros (2009) dedica-se ao exame das séries de vendas no varejo explorando um modelo de mínimos quadrados não linear, em séries de tempo, o LSTAR. Também neste caso opta-se por uma estimativa que é desagregada nas diversas categorias de vendas no varejo. Neste modelo, nota-se que quedas no setor são acompanhadas de um lento processo de recuperação, ao passo que variações positivas muito fortes são efêmeras, não se sustentando, exceto para o segmento de têxteis, calçados e vestuários.

Ao contrário da utilização das séries univariadas, a estratégia preferida por Shousha (2005) é analisar o comportamento das vendas no varejo, confrontando-o com a estrutura a termo das taxas de juros. Paralelamente ao exercício exclusivo com a taxa de juros, realiza-se outro, no qual outras variáveis independentes são acrescidas, tais como taxa de inflação e valores defasados das vendas no varejo.

Fouto (2008) também explora outros fatores macroeconômicos ao analisar o período que vai de 2000 a 2007, e, desse modo, procura estabelecer a dinâmica das vendas no varejo em relação a outras variáveis. Sua escolha é por uma metodologia de séries temporais, em estimações de equações que vão desde estimativas de equações autorregressivas até equações simultâneas, confrontando os resultados obtidos. Em linha geral, seus resultados mostram que, de fato, a renda é um componente importante para explicar o comportamento das vendas no varejo, bem como a trajetória de juros. Entretanto, corroborando um resultado obtido por Campos (2009), o crédito para a pessoa física não se mostrou uma variável importante na determinação das vendas no varejo. 
Portanto, nota-se na literatura um reconhecimento da importância e da necessidade de se entender o comportamento das vendas no varejo. Em outras oportunidades, o acompanhamento das vendas no varejo subsidia o comportamento geral da economia, sendo utilizado, portanto, como indicador antecedente de uma trajetória geral da economia.

Raros trabalhos investigam os elementos macroeconômicos que explicam o desempenho do setor, sobretudo no período recente, tampouco em suas particularidades regionais.

A próxima seçãoapresenta um exercício no qual se busca a determinação das vendas no varejo a partir de uma abordagem regional e através de uma metodologia de regressão em painel.

\section{METODOLOGIA}

Havendo disponibilidade para séries de vendas no varejo em periodicidade mensal e por estados, optou-se por considerar essas informações desagregadas na estimação. Entretanto, como uma das hipóteses do modelo é a ligação entre as vendas no varejo e as condições de emprego e renda, nosso resultado limitou-se a seis estados. Isto porque a Pesquisa Mensal de Emprego (PME), elaborada pelo IBGE, é realizada em seis regiões metropolitanas apenas. Assim, nos limitaremos a examinar os estados da Bahia, de Minas Gerais, de Pernambuco, do Rio de Janeiro, do Rio Grande do Sul e de São Paulo. Note que estão contidos estados relevantes na produção nacional e que somam exatos dois terços (2/3) do PIB brasileiro em 2008 (IBGE, 2010a).

Em outra dimensão, optou-se por se trabalhar o período que se inicia em janeiro de 2004 e se encerra em junho de 2011. Este é um período no qual a economia brasileira cresce sistematicamente, excetuando-se o ano de 2009, e no qual as condições de crédito, taxas de juros, emprego e renda foram extremamente favoráveis. A Tabela 1 resume alguns desses desempenhos.

Deste modo, investigaremos como a renda média real das pessoas ocupadas, a taxa de desemprego, a taxa de juros e a disponibilidade de crédito pessoa física, na modalidade recursos livres, influenciaram as vendas no varejo.

Os dados relativos às vendas no varejo foram coletados junto ao BACEN (2011) e são construídos tendo sua base na média do ano de 2003 e apurados através de pesquisa do IBGE, expressos em números índices. Para a renda real média e a taxa de desemprego os dados estão disponibilizados, respectivamente, em reais de junho de 2011 e na taxa percentual relativa ao desemprego aberto. A taxa de juros Selic corresponde à taxa mensal anualizada, obtida junto ao BACEN (2011), tal como o volume do crédito pessoa física, categoria recursos livres, expresso em milhões de reais de janeiro de 2011. 
Tabela 1 - Indicadores macroeconômicos selecionados, Brasil, 2004 a 2011

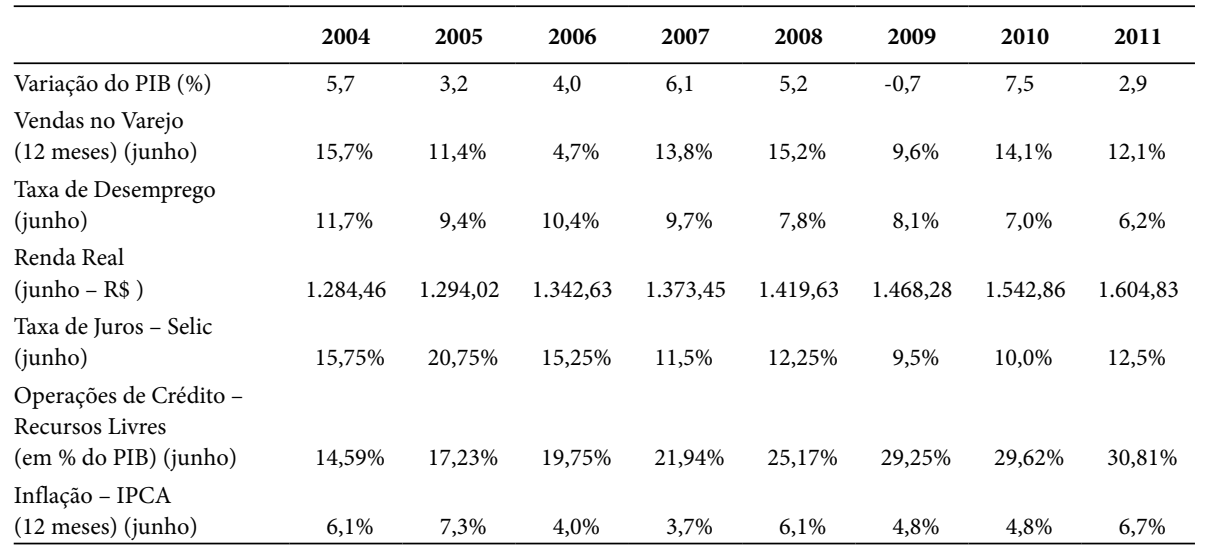

Fonte: Elaboração própria com base em dados do IBGE e Banco Central.

Inicialmente, as variáveis foram transformadas em logaritmo e, na sequência, avaliou-se a correlação entre cada uma das variáveis candidatas a independentes.

Posteriormente, de posse das variáveis definitivamente candidatas a exógenas, testou-se a cointegração em painel destas variáveis, recorrendo-se ao teste de Pedroni (1999, 2004). Dos sete critérios propostos por Pedroni $(1999,2004)$, todos apresentaram o mesmo resultado, ou seja, cointegração em painel. Como consequência, as variáveis ainda que possuam raiz unitária e processo integrado de ordem 1, por serem cointegradas em painel, foram utilizadas em dados originais, convertidos ao logaritmo. Para a taxa de juros Selic, o teste KPSS, proposto por Kwiatkowski et al. (1992) mostrou resultados que confirmam a presença de raiz unitária no período selecionado $^{1}$. Nos anexos A e B resumem-se os resultados obtidos.

A estimação das funções de vendas no varejo, neste artigo, segue a metodologia denominada na literatura Seemingly Unrelated Regressions (SUR), método proposto por Zellner (1962). A razão principal para sua utilização decorre do fato de que as equações que representam as vendas no varejo em cada um dos estados possuem um comportamento empírico relacionado entre si, esperando-se que os fatores tenham comportamentos assemelhados no espaço e período de análise. Não suficiente, as equações apresentam algumas das variáveis independentes em comum. Desse modo, o erro das equações está, provavelmente, correlacionado contemporaneamente, justificando o uso do procedimento. Conforme Zellner (1962), os parâmetros do modelo

1 O teste KPSS mostrou a presença de raiz unitária também. 
são estimados melhor conjuntamente precisamente nesses casos. O teste de BreuschPagan é utilizado para comprovar a relação de dependência entre os resíduos das diferentes equações do sistema.

O modelo SUR pressupõe, segundo Greene (2000), que os erros são homocedásticos e possuem média igual a zero e, ainda, que a covariância entre os erros das equações, ao longo do tempo, seja idêntica a zero. Contudo, existe correlação contemporânea entre os erros das diferentes equações, tendo como consequência a inviabilização da estimação via MQO. Ressalte-se que a utilização de mínimos quadrados generalizados ou estimadores de máxima verossimilhança corrige a violação destes pressupostos. Porém, há duas situações nas quais estimar o modelo SUR via MQO ou MQG resulta idêntico: ou quando todas as correlações contemporâneas são idênticas a zero ou quando as variáveis exógenas são as mesmas para cada uma das equações do sistema. A seguir os pressupostos são detalhados para as especificações do modelo que será utilizado:

$$
\begin{gathered}
\operatorname{Cov}\left(\varepsilon_{\mathrm{It}}, \varepsilon_{\mathrm{It}}\right)=E\left(\varepsilon_{\mathrm{It}}, \varepsilon_{\mathrm{Jt}}\right)=\sigma_{\mathrm{II}} I, J=1,2,3, \ldots, 6 ; \\
E\left(\varepsilon_{\mathrm{It}}\right)=0 \text { para } i=1,2,3, \ldots, 6 ; t=1,2, \ldots, 468 ; \\
\operatorname{Var}\left(\varepsilon_{\mathrm{It}}\right)=E\left(\varepsilon_{\mathrm{It}}\right)^{2}=\sigma_{\mathrm{II}}=\sigma_{\mathrm{I}}^{2} \text { para todo } t=1,2, \ldots, 468 \text { e } I=1,2,3, \ldots, 6 ; \\
\operatorname{Cov}\left(\varepsilon_{\mathrm{It}}, \varepsilon_{\mathrm{Js}}\right)=\operatorname{cov}\left(\varepsilon_{\mathrm{It}}, \varepsilon_{\mathrm{Js}}\right)=0 \text { para todo } T \neq \operatorname{Se} I, J=1,2,3, \ldots, 6 ;
\end{gathered}
$$

Pode-se, a seguir, observar o formato escolhido para cada uma das equações, que contém para cada uma das variáveis independentes.

Enfim, o modelo especificado é composto por variáveis macroeconômicas como candidatas a exógenas e as vendas no varejo como variável endógena.

$\log (\text { Vendas Varejo })_{\mathrm{it}}=\alpha+\beta_{1 \mathrm{i}}{ }^{*} \log (\text { Renda Real })_{\mathrm{it}}+\beta_{2 \mathrm{i}}{ }^{*} \log ($ Taxa

Desemprego $(-3))_{\mathrm{it}}++\beta_{3 \mathrm{i}}{ }^{*} \log (\text { Crédito Pessoa Física }- \text { Recursos Livres })_{\mathrm{it}}+$

$$
+\beta_{4 \mathrm{i}}{ }^{*} \log (\text { Taxa Juros Selic }(-3))_{\mathrm{t}}+A R(1)_{\mathrm{it}}+\varepsilon_{\mathrm{it},}
$$

$\operatorname{Em}(5)$,

$t$ - compreende o período de janeiro de 2004 a junho de 2011;

$i$ - refere-se à indexação por regiões: Bahia, Minas Gerais, Pernambuco, Rio de Janeiro, Rio Grande do Sul e São Paulo;

$\log$ - indica que a variável está transformada para o logaritmo;

Taxa Desemprego(-3) - é a taxa de desemprego defasada em terceira ordem;

Taxa Juros Selic(-3) - é a taxa de juros Selic defasada em terceira ordem;

$A R(1)$ - representa o termo autorregressivo em primeira defasagem; 
A opção por se examinar seis diferentes regiões indica que um painel será estimado, contemplando o período entre 2004 e 2011 e a espacialização do problema.

$\mathrm{Na}$ análise do correlograma, contudo, percebeu-se a necessidade da inclusão do termo autorregressivo de primeira ordem. Ademais, a combinação de defasagens ótimas entre as diferentes variáveis foi testada utilizando-se a minimização da soma dos quadrados dos resíduos como critério. Assim, percebeu-se uma melhor estimativa como expressa em taxa de juros Selic defasada em três meses, tal como a taxa de desemprego. Relativamente à renda real, percebeu-se sua contemporaneidade com relação às vendas no varejo.

\section{RESULTADOS}

Também se testou a possibilidade de efeitos fixos, sendo que estes se mostraram significativos para a estimação. A Tabela 2 contém os resultados da estimação realizada, por estado, e informações adicionais da estatística geral de cada um dos cortes. Nela estão expostos os resultados para o estado de São Paulo: percebe-se que os sinais esperados teoricamente são respeitados, assim como os coeficientes são significativos a $10 \%$ de confiança para o termo autorregressivo de ordem 1. Mais importante, percebe-se inelasticidade para a taxa de juros e a taxa de desemprego, ambas defasadas em três meses, em que uma queda (aumento) na taxa de desemprego há três meses de $1 \%$ resulta em um aumento (queda) nas vendas do varejo de $0,25 \%$, assim como uma queda (aumento) da taxa de juros Selic em $1 \%$ há três meses resulta em um aumento (queda) das vendas no varejo de $0,3 \%$. Contudo, para a renda média real é registrada uma elasticidade expressiva, ou a expansão (decréscimo) em $1 \%$ da renda média real resulta no aumento (queda) de 1,28\% nas vendas no varejo no mesmo mês.

Tabela 2 - Resultados para a estimação em painel, São Paulo, vendas no varejo

\begin{tabular}{lcccc}
\hline \multicolumn{3}{c}{ São Paulo } \\
\hline Variável & Coeficiente & Desvio Padrão & t-Statistic & Prob. \\
\hline Efeito Fixo & $-3,969000$ & & & \\
Selic(-3) & $-0,303188$ & 0,042050 & $-7,210137$ & 0,000000 \\
Taxa de Desemprego (-3) & $-0,250324$ & 0,050452 & $-4,961668$ & 0,000000 \\
Renda Média Real & 1,281127 & 0,110651 & 11,57808 & 0,000000 \\
AR (1) & 0,191305 & 0,106746 & 1,792158 & 0,073700 \\
\hline
\end{tabular}

Fonte: Elaboração própria com base nos resultados da pesquisa.

Na Tabela 3 encontram-se os resultados para o Rio de Janeiro. Entre todas as variáveis, apenas a renda real média mostrou-se significativa. E a intensidade de sua elasticidade é maior do que a percebida em São Paulo, 1,44\%. 
Tabela 3 - Resultados para a estimação em painel, Rio de Janeiro, vendas no varejo

\begin{tabular}{lcccc}
\hline \multicolumn{5}{c}{ Rio de Janeiro } \\
\hline Variável & Coeficiente & Desvio Padrão & t-Statistic & Prob. \\
\hline Efeito Fixo & $-5,742392$ & & & \\
Selic (-3) & $-0,007365$ & 0,043321 & $-0,170013$ & 0,865100 \\
Taxa de Desemprego (-3) & 0,057176 & 0,069796 & 0,819184 & 0,413100 \\
Renda Média Real & 1,445217 & 0,104369 & 13,84713 & 0,000000 \\
AR (1) & 0,000627 & 0,143675 & 0,004366 & 0,996500 \\
\hline
\end{tabular}

Fonte: Elaboração própria com base nos resultados da pesquisa.

Em relação ao comportamento para o estado de Minas Gerais, observa-se, que exceção feita ao termo autorregressivo de primeira ordem, os demais parâmetros são significativos. O padrão elástico volta a ser verificado para a renda média real contemporânea, e inelasticidades são verificadas para a taxa de juros Selic e para a taxa de desemprego, defasadas em três meses.

Tabela 4 - Resultados para a estimação em painel, Minas Gerais, vendas no varejo

\begin{tabular}{lcccc}
\hline \multicolumn{3}{c}{ Minas Gerais } \\
\hline Variável & Coeficiente & Desvio Padrão & t-Statistic & Prob. \\
\hline Efeito Fixo & $-2,977538$ & & & \\
Selic (-3) & $-0,122402$ & 0,042989 & $-2,847283$ & 0,004600 \\
Taxa de Desemprego (-3) & $-0,250324$ & 0,050452 & $-4,961668$ & 0,000000 \\
Renda Média Real & 1,137913 & 0,097442 & 11,67781 & 0,000000 \\
AR (1) & 0,082221 & 0,138412 & 0,594030 & 0,552800 \\
\hline
\end{tabular}

Fonte: Elaboração própria com base nos resultados da pesquisa.

Para o estado do Rio Grande do Sul, um dos resultados mais interessantes refere-se ao fato de que é neste estado que a renda média real apresenta a maior elasticidade, 1,49 , entre as regiões estudadas. Para a taxa de juros, defasada em três meses, não há significância do parâmetro, enquanto para a taxa de desemprego há significância do parâmetro ao nível de 10\%. Os resultados são expostos na Tabela 5.

Tabela 5 - Resultados para a estimação em painel, Rio Grande do Sul, vendas no varejo

\begin{tabular}{lcccc}
\hline \multicolumn{5}{c}{ Rio Grande do Sul } \\
\hline Variável & Coeficiente & Desvio Padrão & t-Statistic & Prob. \\
\hline Efeito Fixo & $-6,182681$ & & & \\
Selic (-3) & $-0,012550$ & 0,050278 & $-0,249624$ & 0,803000 \\
Taxa de Desemprego (-3) & $-0,084659$ & 0,050387 & 1,680169 & 0,093600 \\
Renda Média Real & 1,496586 & 0,109805 & 13,62943 & 0,000000 \\
AR (1) & 0,290693 & 0,118898 & 2,444892 & 0,014800 \\
\hline
\end{tabular}

Fonte: Elaboração própria com base nos resultados da pesquisa. 
Para o estado da Bahia, conforme a Tabela 6, a renda média real mostrou-se elástica, repetindo o padrão dos demais estados, ao passo que a taxa de juros Selic e a taxa de desemprego, defasadas em três meses, são significantes, mas inelásticas. Finalmente, a Tabela 7 apresenta os resultados para o estado de Pernambuco, no qual se percebe a menor elasticidade-renda dentre os estados pesquisados.

Tabela 6 - Resultados para a estimação em painel, Bahia, vendas no varejo

\begin{tabular}{lcccc}
\hline \multicolumn{1}{c}{ Variável } & Coeficiente & Desvio Padrão & t-Statistic & Prob. \\
\hline Efeito Fixo & $-3,690379$ & & & \\
Selic (-3) & $-0,098124$ & 0,048778 & $-2,011666$ & 0,044800 \\
Taxa de Desemprego (-3) & $-0,235271$ & 0,084410 & $-2,787255$ & 0,005500 \\
Renda Média Real & 1,314062 & 0,112213 & 11,710450 & 0,000000 \\
AR (1) & 0,095034 & 0,109977 & 0,864122 & 0,388000 \\
\hline
\end{tabular}

Fonte: Elaboração própria com base nos resultados da pesquisa.

Tabela 7 - Resultados para a estimação em painel, Pernambuco, vendas no varejo

\begin{tabular}{lcccc}
\hline \multicolumn{1}{c}{ Variável } & Coeficiente & Desvio Padrão & t-Statistic & Prob. \\
\hline Efeito Fixo & $-1,874791$ & & & \\
Selic (-3) & $-0,218696$ & 0,049280 & $-4,437825$ & 0,000000 \\
Taxa de Desemprego (-3) & $-0,215817$ & 0,053719 & $-4,017540$ & 0,000100 \\
Renda Média Real & 1,066861 & 0,075255 & 14,176570 & 0,000000 \\
AR (1) & 0,327318 & 0,086037 & 3,804394 & 0,000200 \\
\hline
\end{tabular}

Fonte: Elaboração própria com base nos resultados da pesquisa.

Como percepção geral nota-se que a renda média real contemporânea é um fator predominante para explicar o comportamento das vendas no varejo no Brasil. Isto é condizente, sobretudo, com um perfil de consumo de baixo e médio poder aquisitivo, característico da população brasileira, cuja propensão marginal a poupar é baixa. Corrobora esta percepção o fato de que a taxa de desemprego, em menor grau, demonstra-se uma variável importante nesta determinação, exceto para o Rio de Janeiro, confirmando que a sensação de renda estável é algo determinante na disposição à aquisição de produtos no varejo. Ademais, como esperado, a taxa de juros é um componente importante na determinação das vendas no varejo, com uma relação negativa, conforme previsto na teoria e reforçando seu papel contra-cíclico, exceto para o Rio de Janeiro e o Rio Grande do Sul. Deve-se notar que as duas últimas variáveis apresentaram uma reação que se estende por um trimestre até impactar as vendas no varejo.

Nos anexos C e D são apresentados os testes para a estacionariedade dos resíduos de cada estado, ou cross-section. Percebe-se que atendem às propriedades desejadas. 


\section{COMENTÁRIOS FINAIS}

Avaliando-se os recentes anos de crescimento econômico do Brasil, período que se inicia no ano de 2004, pode-se perceber alguns comportamentos associados a vendas no varejo. O setor possui certa importância no cômputo do produto interno bruto e é um importante empregador.

Como resultado geral, percebeu-se que as vendas no varejo são condicionadas por três fatores macroeconômicos, quais sejam: taxa de juros, taxa de desemprego, renda média real. Percebe-se que, para os seis estados pesquisados, no período entre janeiro de 2004 e junho de 2011, o comportamento das vendas no varejo foi idêntico para essas regiões, com a renda média real contemporânea demonstrando-se elástica para vendas no varejo, ao passo que a taxa de desemprego e a taxa de juros demonstraram-se coeficientes inelásticos para vendas no varejo com melhor ajustamento quando se considera a defasagem de três trimestres. Entretanto, a intensidade das variáveis dos efeitos nos estados diferencia-se.

Assim, percebe-se, através de uma estimação em painel, que, fundamentalmente, o comportamento do setor depende sensivelmente do mercado de trabalho e das condições que este apresenta, criando a disposição para o gasto no varejo. O resultado pode ser aprimorado em trabalhos futuros, nos quais se avaliariam outros estados. Ademais, seria interessante conhecer como esse comportamento poderia se alterar ou manter-se em períodos de desaceleração econômica.

\section{REFERÊNCIAS}

ALMEIDA, F. C.; PASSARI, A. F. L. Previsão de vendas no varejo por meio de redes neurais. Revista de Administração, v. 41, n. 3, p. 257-272, 2006.

De ANGELO, C. F.; ZWICKER, R.; FOUTO, N. M. M. D.; LUPPE, M. R. Temporal series and neural networks: a comparative analysis of techniques in the Brazilian retail sales forecast. BBR - Brazilian Business Review, v. 8, n. 2, p. 1-21, 2011.

ARAUJO, V. L. Preferência pela liquidez dos bancos públicos no ciclo de expansão do crédito no Brasil: 2003-2010. In: Encontro Nacional de Economia Política, 16, Uberlândia, 2010. Anais... Uberlândia, MG: 2010.

BANCO CENTRAL DO BRASIL (BACEN). Sistema Gerenciador de Séries Temporais. 2011. Disponível em: <http://www4.bcb.gov.br/pec/series/port/aviso.asp>. Acesso em: 15 jun. 2015

BRONZI, T. P.; SCARPEL, R. A. Modelo de previsão do nível de atividade via método da composição dos especialistas locais. In: Encontro de Iniciação Científica do Instituto Tecnológico da Aeronáutica, 16, São José dos Campos, SP, 2010.

CAMPOS, J. C. Os impactos da conjuntura macroeconômica sobre o comércio no Brasil. Uma análise empírica dos grandes varejistas. Dissertação de Mestrado, Programa de Pós-Graduação em Finanças e Economia Empresarial, Fundação Getúlio Vargas, Rio de Janeiro, 2009. 
CAPITANI, L. M.; SCARPEL, R. A.; DOTTI, E. Identificação do número mínimo-ideal de indicadores econômicos por geração de agrupamentos de séries temporais. In: Encontro do Simpósio Brasileiro de Pesquisa Operacional, 62, Bento Gonçalves, RS, 2010. Anais... Bento Gonçalves: SOBRAPO, 2010.

COSTA, F. N.; DE DEOS, S. S.; DE BRITO, J. V. meta inflacionária, juros e preços no varejo brasileiro. Revista de Economia Política, v. 21, n. 4 (84), p. 93-111, 2001.

FALEIROS, J. P. M. Comércio varejista: uma análise do comportamento do volume de vendas sob uma perspectiva nãolinear. Revista do BNDES, n. 32, p. 157-182, 2009.

FUNDO MONETÁRIO INTERNACIONAL (FMI). Data and Statistics. 2011. Disponível em: <http://www.imf.org/external/data.htm>. Acesso em: 15 jun. 2015.

FOUTO, N. M. M. Mercado de consumo brasileiro: evolução e determinantes do volume de vendas. Uma análise do período pós-real. São Paulo: Tese de Doutorado, Programa de Pós-Graduação em Administração, Faculdade de Economia e Administração, Universidade de São Paulo, 2008.

INSTITUTO BRASILEIRO DE GEOGRAFIA E ESTATÍSTICA (IBGE). Contas Regionais do Brasil 2004-2008. Rio de Janeiro: IBGE, 2010a. Disponível em: <http://www.ibge.gov.br/ home/estatistica/economia/contasregionais/2008/publicacao2008.pdf >. Acesso em: 15 jun. 2015.

INSTITUTO BRASILEIRO DE GEOGRAFIA E ESTATÍSTICA (IBGE). Pesquisa Anual de Comércio. Rio de Janeiro: IBGE, 2010b. Disponível em: <http://www.ibge.gov.br/home/estatistica/economia/comercioeservico/pac/2009/defaulttabpdf.shtm>. Acesso em: 15 jun. 2015.

INSTITUTO BRASILEIRO DE GEOGRAFIA E ESTATÍSTICA (IBGE). Pesquisa Mensal de Emprego. Rio de Janeiro: IBGE, 2011a. Disponível em: <http://www.ibge.gov.br/home/estatistica/indicadores/trabalhoerendimento/pme_nova/defaulttab2.shtm>. Acesso em: 15 de jun. 2015.

INSTITUTO BRASILEIRO DE GEOGRAFIA E ESTATÍSTICA (IBGE). Sistema de Contas Trimestrais. Rio de Janeiro: IBGE, 2011b. Disponível em: <http://www.ibge.gov.br/home/estatistica/indicadores/pib/defaultcnt.shtm>. Acesso em: 15 de jun. 2015.

KWIATKOWSKI, D; PHILLIPS, P. C. B; SCHMIDT, P; SHIN, Y. Testing the Null Hypothesis of Stationarity against the Alternative of a Unit Root. Journal of Econometrics, v. 54, p. 159-178, 1992.

MODENESI, A. M. Convenção e rigidez na política monetária: uma estimativa da função de reação do BCB - 2000-2007. Texto para Discussão, Rio de Janeiro, n. 1351, 2008.

PASQUOTTO, J. L. D. Previsão de séries temporais no varejo brasileiro: uma investigação comparativa da aplicação de redes neurais recorrentes de Elman. Tese de Doutorado, Programa de Pós-Graduação em Administração, Faculdade de Economia e Administração, Universidade de São Paulo, 2010.

PEDRONI, P. Critical values for cointegration tests in heterogeneous panels with multiple regressors. Oxford Bulletin of Economics and Statistics, special edition, p. 653-670, 1999.

PEDRONI, P. Panel cointegration: asymptotic and finite sample properties of pooled time series tests with an application to the PPP hypothesis. Econometric Theory, p. 597-625, 2004. 
ROSTOW, W. W. Etapas do desenvolvimento econômico. México: Fondo de Cultura Económica, 1970.

SHOUSHA, S. Estrutura a termo da taxa de juros e dinâmica macroeconômica do Brasil. Dissertação de Mestrado, Programa de Pós-Graduação em Economia, Departamento de Economia, Pontifícia Universidade Católica do Rio de Janeiro, 2005.

ZELLNER, A. An efficient method of estimating seemingly unrelated regressions and tests for aggregation bias. Journal of the American Statistical Association, n. 57 p. 348-368, jun. 1962. 


\section{ANEXO A}

Tabela 8 - Teste de raiz unitária para as séries de dados - janeiro de 2004 a junho de 2011 - valores críticos e intervalo de aceitação

\begin{tabular}{lcc}
\hline Série & KPSS & Decisão \\
\cline { 2 - 3 } Vendas Varejo BA & Ho:Estacionaridade & I (1) \\
Vendas Varejo MG & 1,295543 & $\mathrm{I}(1)$ \\
Vendas Varejo PE & 1,327893 & $\mathrm{I}(1)$ \\
Vendas Varejo RJ & 1,304321 & $\mathrm{I}(1)$ \\
Vendas Varejo RS & 1,283140 & $\mathrm{I}(1)$ \\
Vendas Varejo SP & 1,227066 & $\mathrm{I}(1)$ \\
Renda Real BA & 1,343587 & $\mathrm{I}(1)$ \\
Renda Real MG & 1,295384 & $\mathrm{I}(1)$ \\
Renda Real PE & 1,315106 & $\mathrm{I}(1)$ \\
Renda Real RJ & 0,934692 & $\mathrm{I}(1)$ \\
Renda Real RS & 1,285316 & $\mathrm{I}(1)$ \\
Renda Real SP & 1,267529 & $\mathrm{I}(1)$ \\
Desemprego BA & 1,232606 & $\mathrm{I}(1)$ \\
Desemprego MG & 1,167446 & $\mathrm{I}(1)$ \\
Desemprego PE & 1,175981 & $\mathrm{I}(1)$ \\
Desemprego RJ & 0,964541 & $\mathrm{I}(1)$ \\
Desemprego RS & 1,149415 & $\mathrm{I}(1)$ \\
Desemprego SP & 1,091064 & $\mathrm{I}(1)$ \\
Taxa de Juros SELIC & 1,018414 & $\mathrm{I}(1)$ \\
\hline
\end{tabular}

Fonte: Elaboração própria com base nos resultados da pesquisa. 


\section{ANEXO B}

Tabela 9 - Teste de cointegração em painel de Pedroni Hipótese nula: não há cointegração Seleção: Critério de Akaike com defasagem máxima de três períodos

\begin{tabular}{lcccc}
\hline \multicolumn{5}{c}{ Hipótese alternativa: coeficientes autorregressivos comuns (within-dimension) } \\
\hline \multicolumn{7}{c}{ Estatística } & Prob. & Estatística Ponderada & Prob. \\
\hline Painel V-Estatística & 4,808452 & 0.0000 & 4,225713 & 0.0000 \\
Painel rho-Estatística & $-17,56221$ & 0.0000 & $-17,18613$ & 0.0000 \\
Painel PP-Estatística & $-13,34058$ & 0.0000 & $-13,32051$ & 0.0000 \\
Painel ADF-Estatística & $-5,363912$ & 0.0000 & $-6,443139$ & 0.0000 \\
\hline Hipótese alternativa: coeficientes autorregressivos individuais (between-dimension) & \multicolumn{2}{c}{ Prob. } \\
\hline \multicolumn{7}{c}{ Estatística } & 0.0000 \\
Grupo rho-Estatística & $-18,62786$ & 0.0000 \\
Grupo PP-Estatística & $-16,30634$ & 0.0000 \\
Grupo ADF-Estatística & $-6,162921$ &
\end{tabular}

Fonte: Elaboração própria com base nos resultados da pesquisa. 
ANEXO C

TESTE DE ESTACIONARIEDADE DOS RESÍDUOS

Tabela 10 - Valores do teste de raiz unitária para os resíduos das equações

\begin{tabular}{lcccc}
\hline \multirow{1}{*}{ Série } & Dickey Fuller & Phillips Perron & K-P-S-S & \multirow{2}{*}{ Decisão } \\
\cline { 2 - 4 } & H.o:RaizUnitária & Ho:RaizUnitária & Ho:Estacionaridade & \\
\hline Resíduos BA & $-8,110587$ & $-8,116525$ & 0,107457 & Estacionário \\
ResíduosMG & $-9,285690$ & $-9,337804$ & 0,088570 & Estacionário \\
ResíduosPE & $-7,477015$ & $-7,583330$ & 0,282168 & Estacionário \\
ResíduosRJ & $-5,006730$ & $-8,583155$ & 0,038638 & Estacionário \\
ResíduosRS & $-2,138060$ & $-7,158006$ & 0,068339 & Estacionário \\
ResíduosSP & $-1,453967$ & $-8,710485$ & 0,268198 & Estacionário \\
\hline
\end{tabular}

Fonte: Elaboração própria com base nos resultados da pesquisa. 
ANEXO D

Figura 1 - Gráfico dos resíduos da estimação em painel
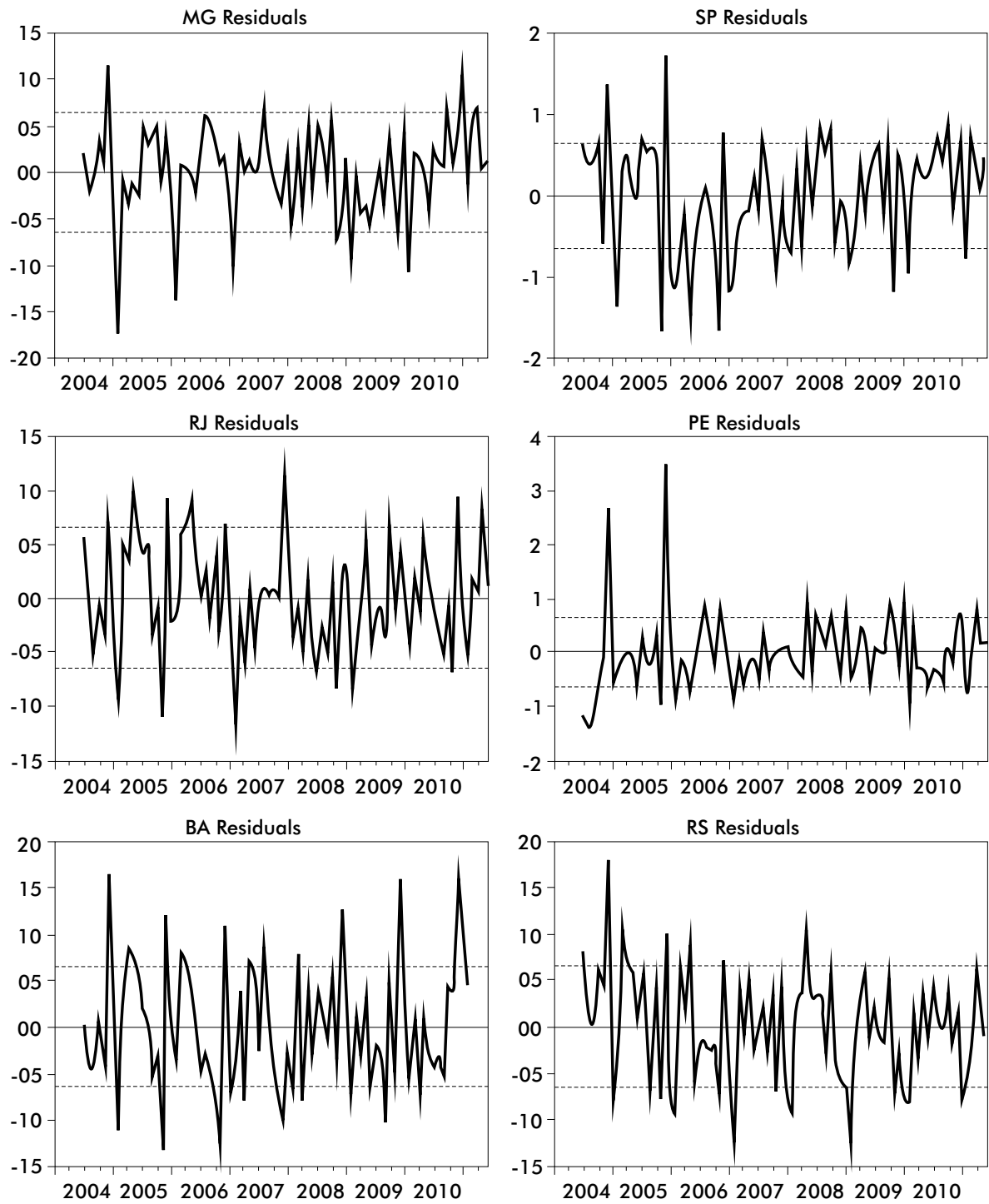

Fonte: Elaboração própria com base nos resultados da pesquisa. 